\title{
The Effects of Speed Uncertainty on a Separation Assurance Algorithm
}

\author{
Todd A. Lauderdale* \\ NASA Ames Research Center, Moffett Field, CA, 94035
}

\begin{abstract}
Trajectory prediction is central to separation assurance, because it is necessary to accurately predict where an aircraft will be in the future to avoid losses of separation. In any system in the field there will always be uncertainty associated with trajectory prediction. This uncertainty is a product of many different sources including wind prediction errors, pilot intent, surveillance errors, navigation errors and aircraft weight. The purpose of this study is to parametrically analyze the effects of aircraft speed errors on conflict detection and to analyze the performance of a conflict resolution algorithm when these speed errors are present. Results show that a speed error of $\pm 10 \%$ of the cruise speed can result in about $30 \%$ of the conflicts not being detected ten minutes before the loss of separation. It can also result in $\mathbf{5 0 \%}$ more resolutions being performed to maintain separation.
\end{abstract}

\section{Introduction}

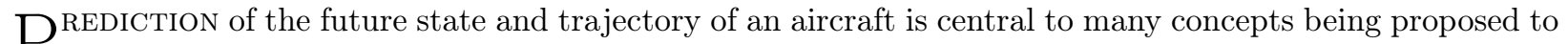
improve the efficiency and safety of the air transportation system. Accurate trajectory prediction plays an especially important role in automated concepts for separation assurance because it allows for reliable prediction of future losses of separation and for generation of safe resolution trajectories. Unfortunately, there will always be errors in any predicted trajectory since the model used to generate this prediction is only an approximation of the aircraft and the environment through which it is flying (Figure 1). Independent causes of the error of any trajectory prediction include, among many other things, a lack of accurate knowledge of the winds aloft, only partial information about how a pilot will execute any given maneuver, errors in the surveillance of the aircraft position and velocity, and inaccurate information about the weight of the aircraft.

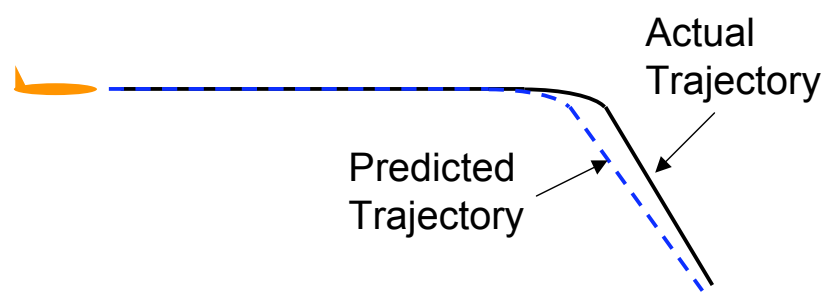

Figure 1. A schematic showing how a predicted trajectory might vary from an actual flown trajectory.

Since there is no way to predict the future state of an aircraft with zero error, automation tools designed to perform safety-critical functions will often sacrifice some airspace throughput in order to maintain a high level of safety. Separation assurance systems are no exception to this in that they often use limited look-ahead times, additional spatial buffers, and probabilistic analyses to account for trajectory prediction uncertainty. These added buffers and limited look-ahead times come with a commensurate reduction in airspace capacity and throughput. ${ }^{1}$

In the past there have been studies of the accuracy of specific trajectory predictors as compared to flight data $^{2,3}$ as well as the effects of this accuracy on the performance of some decision support tools. ${ }^{4,5}$ These are, in general, point design studies conducted to evaluate how a specific design performs under specific types

*Aerospace Engineer, NASA Ames Research Center, AIAA Member

$$
1 \text { of } 11
$$


and levels of uncertainty. There have also been analytical analyses of the effects of uncertainty, ${ }^{6}$ but these make assumptions about the types of conflicts encountered.

The purpose of this study is to perform a more general, parametric evaluation of the performance of a separation assurance algorithm under a wide range of values of a single type of trajectory prediction error. This study was conducted using a realistic mix of aircraft conflicts derived from the routes flown by aircraft in today's system. This broad study allowed for a parametric assessment of the trade-offs required between safety and efficiency for specific levels of uncertainty. The converse question can be answered as well: what is the maximum trajectory prediction error for a desired performance level?

\section{Simulation Environment}

For this study, the Airspace Concept Evaluation System (ACES) ${ }^{7}$ was used to simulate the National Airspace system (NAS). The separation assurance algorithm evaluated in this simulation environment was the Advanced Airspace Concept (AAC) ${ }^{8}$ Autoresolver. $^{9}$

\section{A. ACES}

ACES is a non-real time, gate-to-gate simulation of the NAS that creates four-degree-of-freedom trajectories of aircraft based on the aircraft performance models from the Base of Aircraft Data (BADA) from departure fix to arrival fix. The routes and departure times for the aircraft in the simulation are derived from the actual routes flown on a specific day in the NAS.

In the base implementation of ACES the future predictions of all aircraft states have zero error as compared to the trajectory that the simulated aircraft flies because both the trajectory predictor and the simulated aircraft use the same equations of motion and environmental data. Section III discusses modifications made to the trajectory prediction capability of ACES for this study.

\section{B. AAC Autoresolver}

The Advanced Airspace Concept is a concept for automating separation assurance in the future including multiple layers of separation assurance for increased reliablilty. One component of AAC is a strategic problem-solving tool known as the Autoresolver which is responsible for strategic separation assurance as well as weather avoidance and arrival metering, ${ }^{9,10}$ although for this study the focus is only on the separation assurance function. The look-ahead time used to solve these problems is between 20 and 2 minutes before the occurrence of any of these problems. This algorithm was originally developed in the ACES environment taking full advantage of the zero-error trajectory prediction available, and many studies of the effectiveness of this algorithm in the zero uncertainty environment have been performed. ${ }^{11}$ It has also been integrated with and evaluated in other NAS simulations which have non-zero trajectory prediction errors. ${ }^{12-14}$

For strategic conflict detection and resolutions predicted trajectories are created for all aircraft periodically. These trajectories are created assuming the intent of the aircraft including the cruise speed, future waypoints, and cruise altitude is known. These predicted trajectories are compared against each other pointwise to identify future possible losses of separation.

The AAC Autoresolver uses an iterative approach to resolve all of these conflicts. The algorithm attempts to generate many different types of conflict-free resolutions for any conflict. The set of conflict-free resolutions can include multiple horizontal, multiple vertical, and multiple speed resolutions. After the resolution trajectories have been generated, the successful resolution with the minimum delay is chosen for implementation.

There are many different parameters of the Autoresolver that can be adjusted to deal with uncertainty including a detection buffer, a resolution buffer, a look-ahead time for conflicts, and a look-ahead time for resolutions. The detection buffer (Figure 2(a) is defined as the added radius for the detection range above the required separation radius. Likewise, the resolution buffer (Figure 2(b) is the additional radius outside the detection range, including the detection buffer, that a resolution attempt must be clear of in order for it to be considered successful. This resolution buffer is designed to reduce the possibility of a conflict reoccuring. If necessary, additional types of buffers could be added for detection and resolution to mitigate climb or descent uncertainty similar to the work presented by Thipphavong. ${ }^{15}$

Increasing the detection buffer can lead to fewer missed conflicts and more separation between aircraft but may result in more false alerts. Similarly, increasing the resolution buffer can result in fewer resolutions 
Detection

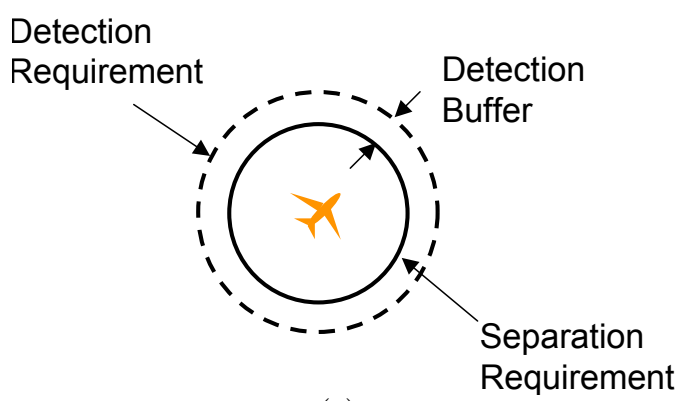

(a)

\section{Resolution}

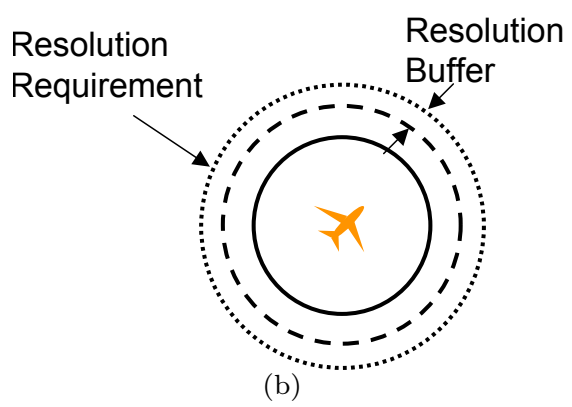

Figure 2. An illustration of the required separation standard as well as (a) the detection requirement including buffer and (b) the resolution requirement including buffer.

that need to be redone because of trajectory errors, but a larger resolution buffer causes greater average delays. ${ }^{1}$ Another trajectory-error mitigation methodology is to limit the look-ahead time for conflict detection. Limiting this time can reduce the number of false alerts. One focus of this study is to assess these trade-offs in buffer sizes and conflict resolution look-ahead times.

\section{Trajectory Prediction and Perturbation}

ACES is a powerful tool for understanding the effects of uncertainty on separation assurance because the same trajectory generator is used to simulate the aircraft and to perform trajectory prediction for conflict detection and resolution. This allows for the uncertainty to be varied smoothly from zero to any reasonable value.

In ACES, each time a conflict detection and resolution cycle is initiated, an exact copy of the trajectory generator used to model the simulated aircraft is made and used to create the trajectory prediction for that aircraft. For the current study, two copies of the trajectory generator were created; one of them was perturbed while the other was not. The unperturbed trajectory generator provided a base of perfect knowledge of how the aircraft would maneuver, while the perturbed trajectory generator was used for conflict detection and resolution.

For this study, the perturbation applied to the trajectory generator was an increase in the cruise speed for the cruise portion of the trajectory. In the real system, the ground-speed uncertainty would primarily be caused by wind prediction errors, so it would not be uniform for all aircraft. However, to facilitate understanding and insight into the results of the study, the same speed perturbation was applied to all trajectory generators in the system for this experiment, and no winds were simulated.

Figure 3 shows the details of an actual flown trajectory and the perturbation applied to a trajectory generated for conflict detection. The cruise speed error of the aircraft in this figure was $+5 \%$, as shown in Figure 3(a). The descent speed profile was not perturbed, but since the aircraft is cruising faster it reaches the top-of-descent point sooner in the perturbed trajectory than on the flown trajectory (Figure 3(b)]. Figure 3(c) shows that the lateral path flown by the aircraft is not affected by this speed perturbation, but the along-track error is non-zero as demonstrated by the data in Figure $3(\mathrm{~d})$. The perturbed trajectory reaches any given latitude sooner than the actual aircraft.

For this study, a $+10 \%$ error indicates that the conflict detection and conflict resolution trajectories were generated with a cruise speed $10 \%$ faster than the actual aircraft was flying. A $-10 \%$ error indicates that the predicted trajectories where generated with a cruise speed $10 \%$ slower than the actual trajectory. As noted before, all aircraft are perturbed by the same amount.

\section{Experiment Setup}

To study the effects of the conflict detection and conflict resolution buffer sizes for different levels of speed uncertainty, the problem was decomposed into two related studies: a conflict detection study and a conflict 


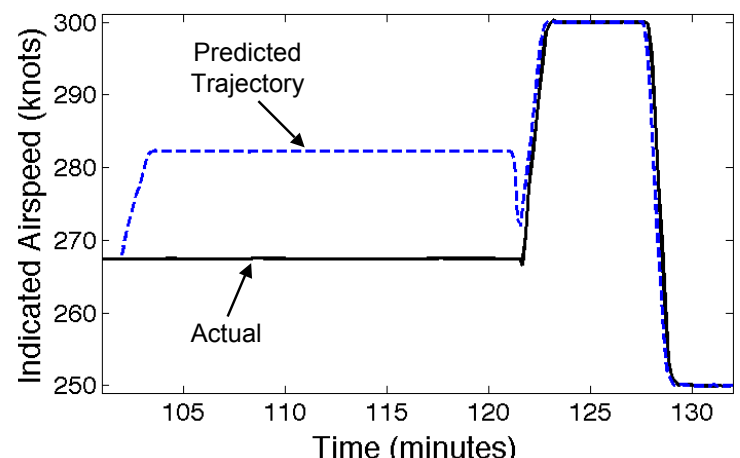

(a)

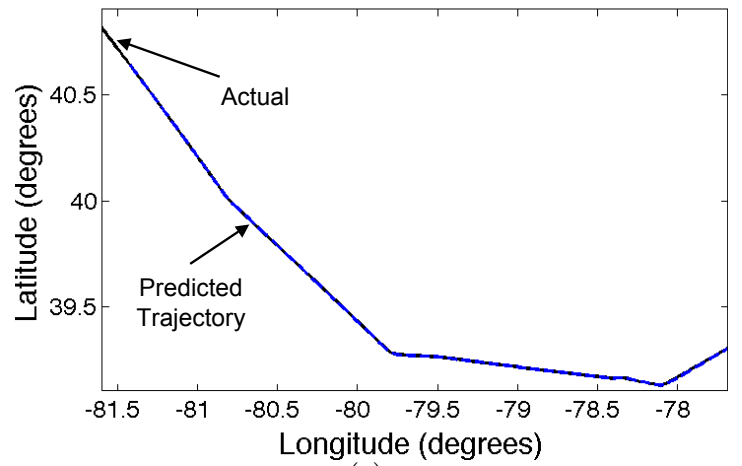

(c)

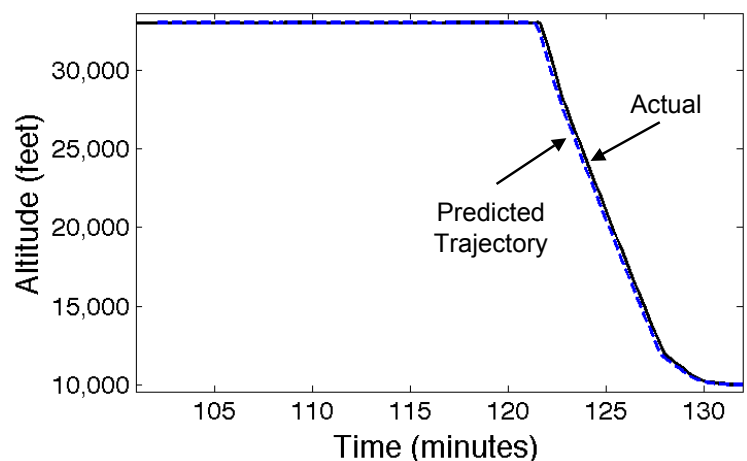

(b)

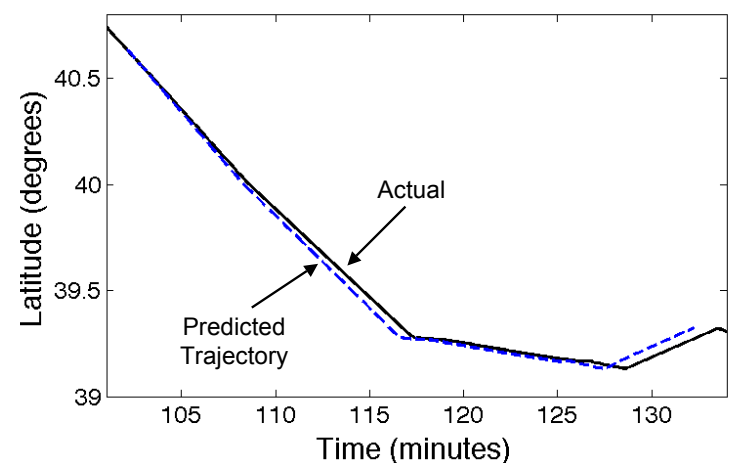

(d)

Figure 3. An example of an actual trajectory and a predicted trajectory including a $5 \%$ speed perturbation. Shown are (a) the speed, (b) the altitude, (c) the lateral path, and (d) the latitude as a function of time.

resolution study.

\section{A. Conflict Detection}

The purpose of the conflict detection study was to determine the effectiveness of the conflict detection algorithm as a function of the speed uncertainty and the conflict detection buffer. To accomplish this, ACES was run without the Autoresolver, the conflict detection buffer was set to 0,1 , or 2 nautical miles, and the cruise speed error was varied.

The major source of cruise speed uncertainty in the system is wind uncertainty. A study of wind forcast errors showed that wind speed prediction errors can be up to 15 knots. ${ }^{16}$ This is a little less than $5 \%$ of a nominal aircraft cruise speed. The aircraft speed errors were set to $-10,-5,0,5$, or 10 percent of the cruise speed in this study to explore nominal and extreme trajectory speed error scenarios.

A flight data set with three hours of traffic at current demand levels based on recorded data was simulated for the entire NAS. The conflict detection function was run for all traffic across the entire airspace once every minute in the simulation, and each time both the perturbed and unperturbed trajectory predictions were used for conflict detection. The unperturbed trajectories allowed for completely accurate knowledge of the existence and geometric and temporal properties of a conflict. The perturbed trajectories simulated how a trajectory prediction system with that level of speed error would function, and the results of the conflict detection using the perturbed trajectory were compared to the accurate conflict detection.

The schematics in Figure 4 show how the two trajectories created for each aircraft in a conflict detection cycle were used. Note that, while this study is interested in the effects of speed errors, these figures represent horizontal errors for ease of representation. Figure 4(a) shows how a missed alert is defined for this study. In this schematic the perturbed and unperturbed trajectories are shown for both aircraft. Conflict detection performed on the unperturbed trajectories finds that the aircraft are currently in conflict, but the perturbed trajectories do not capture this conflict, resulting in a missed alert. Figure 4(b) shows a corresponding false alert, where the perturbed trajectories indicate a conflict, but the actual trajectories do not cause a loss of 
separation.

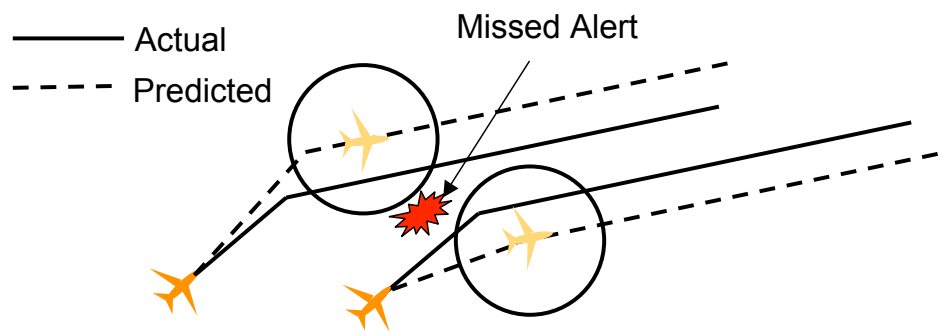

(a)

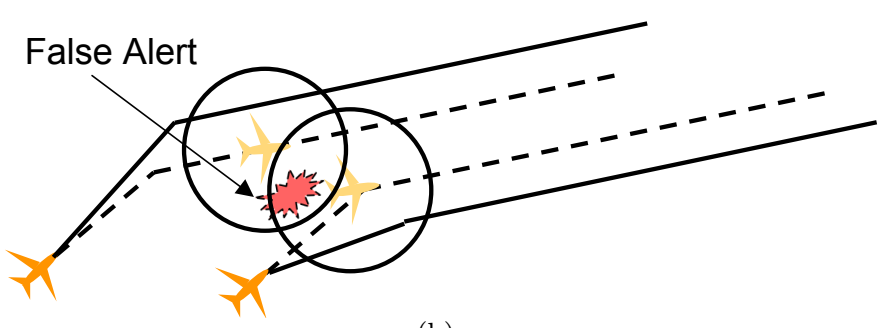

(b)

Figure 4. A schematic show how trajectory prediction errors can lead to (a) missed alerts and (b) false alerts.

\section{B. Conflict Resolution}

The conflict resolution study helps to determine the required size of the conflict resolution buffer. For this study, the AAC Autoresolver was active and resolving conflicts with a look-ahead time of 8 minutes for primary conflicts. All proposed resolutions were required to be clear of conflicts for at least 12 minutes. These two parameters are related to the uncertainty of the trajectory prediction, but for this study they were held fixed. The conflict detection buffer was fixed at $1 \mathrm{nmi}$. The conflict resolution buffer was varied to be either 0,1 , or $2 \mathrm{nmi}$. The same flight demand set was used for both the conflict detection study and the conflict resolution study, and the speed error was also varied to be $-10,-5,0,5$, or 10 percent. The 15 ACES runs provided data about how the conflict resolution buffer affects the efficiency of the system under varying trajectory speed errors.

\section{Results}

The two different studies were performed to provide a parametric understanding of the effects of the trajectory uncertainty mitigation buffers on both conflict detection and conflict resolution performance. The results of these two studies are presented in the next two sections.

\section{A. Conflict Detection}

As described in Section IV] conflict detection was performed for 15 different combinations of speed error and conflict detection buffer size. The actual properties of conflicts were recorded using unperturbed trajectories. The perturbed trajectories are used to identify predicted conflicts, and the differences between these two conflict detection lists provides the accuracy of the conflict prediction under the given trajectory speed error.

Figure 5 shows the conflict detection accuracy in terms of the percentage of conflicts that were not detected using the perturbed trajectories. Results for the three different conflict detection buffer sizes are presented as a function of time to first loss of separation for speed errors of -5 (Figure 5(a)) and -10 percent (Figure 5(b)]. For both cases the number of missed detections decreases with increasing buffer size and shorter time to loss of separation. The percentage of alerts missed with $-10 \%$ speed errors is greater than the percentage of missed alerts with the smaller speed error. Interestingly, for buffer sizes up to $2 \mathrm{nmi}$ there are still some missed alerts even as close as two minutes to loss of separation. This suggests that with speed uncertainty present, a spacial buffer alone may not be sufficient to guarantee that all conflicts will be identified with sufficient time to resolve them.

$$
5 \text { of } 11
$$




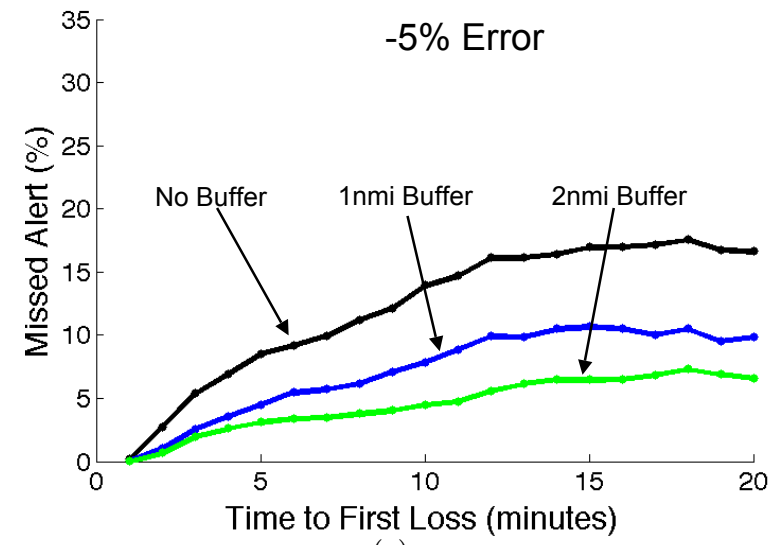

(a)

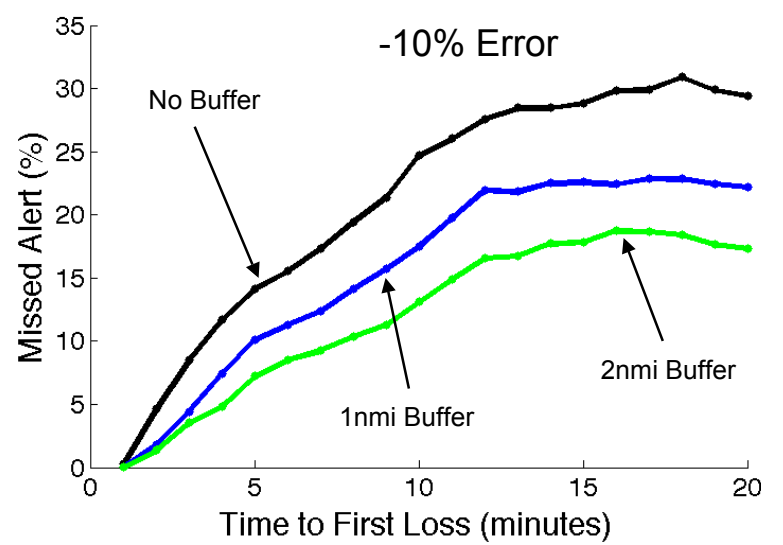

(b)

Figure 5. The percentage of conflicts which are not predicted for different conflict detection buffers as functions of time to first loss for (a) a $-5 \%$ speed error and (b) a $-10 \%$ speed error.

Similar data is presented in Figure 6, but instead of holding the speed error constant the detection buffer is held constant. Figure 6(a) shows how the missed conflicts vary under all the studies speed errors for no conflict detection buffer, while Figure 6(b) shows these data for a 2 nmi buffer. As expected, there are fewer missed alerts for larger buffer sizes and for lower values of speed error. At $+10 \%$ speed error the percentage of missed conflicts does not drop to 0 as the time to first loss decreases. This is a result of failures of the trajectory generator to create a trajectory. The $+10 \%$ speed increase placed the cruise speed outside the performance envelope of the aircraft. When this occurs, the trajectory generator is not able to create a trajectory which meets all of the constraints and returns a failure instead.

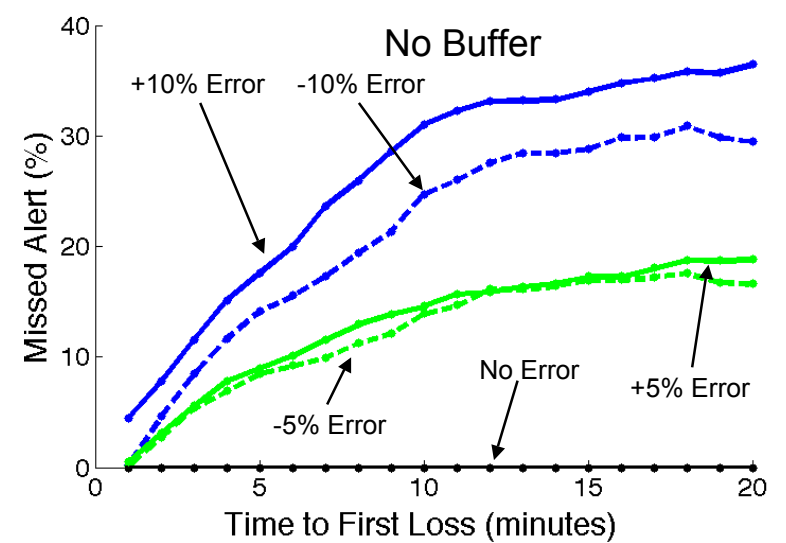

(a)

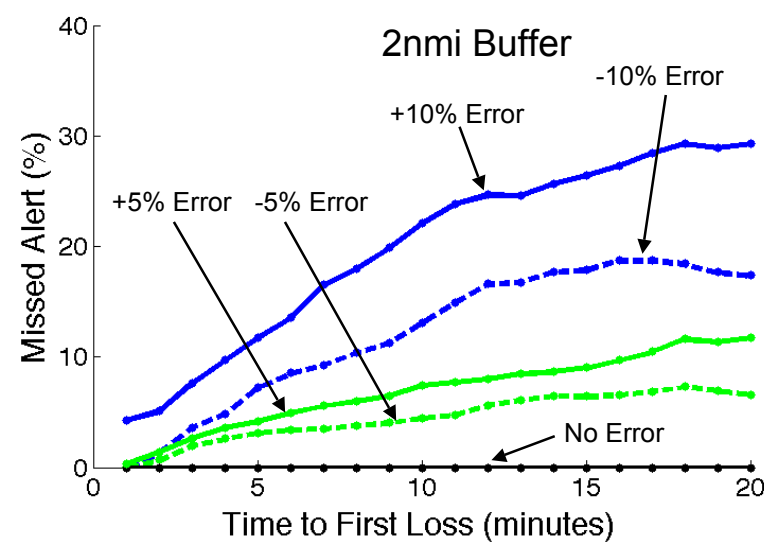

(b)

Figure 6. The percentage of conflicts which are not predicted for different speed errors as functions of the time to first loss for (a) no detection buffer and (b) a 2 nmi detection buffer.

Similar to the missed conflicts, the number of false alerts can be calculated at each conflict detection iteration by matching the true conflicts with the predicted conflicts. Figure 7 shows the percentage of false alerts for the different detection buffer sizes as functions of time to first loss of separation. In Figure 7(a) the speed error is held constant at $-5 \%$. The percentage of false alerts increases with increasing buffer size. Unlike missed alerts, false alerts do not converge to zero when approaching the time of first loss except for the zero buffer case because this is a geometric property of the detection buffer area. The percentage of false alerts increases slowly as a function of time to first loss for $-5 \%$ speed error, but for $-10 \%$ error (Figure $7(\mathrm{~b})$ the percentage of false alerts is a stronger function of the time to first loss.

The percentage of false alerts observed while holding the detection buffer constant is presented in Figure 8. For no buffer (Figure 8(a) , the percentage of false alerts is a strong function of both the time to first loss 


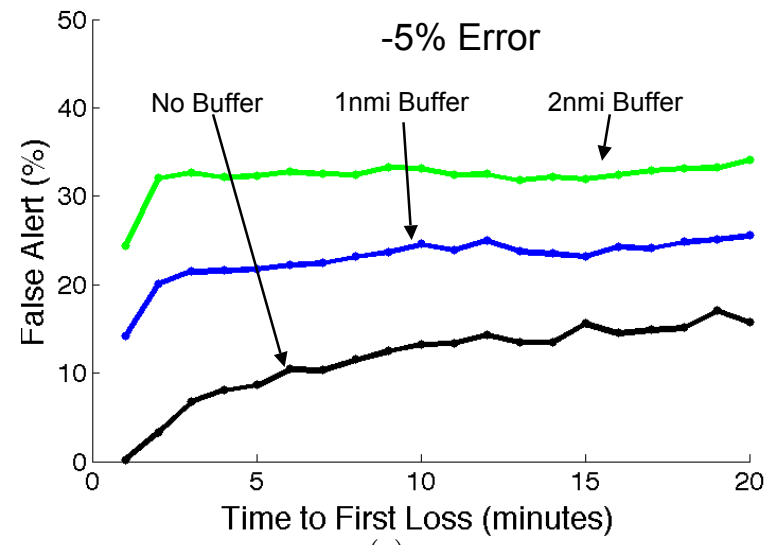

(a)

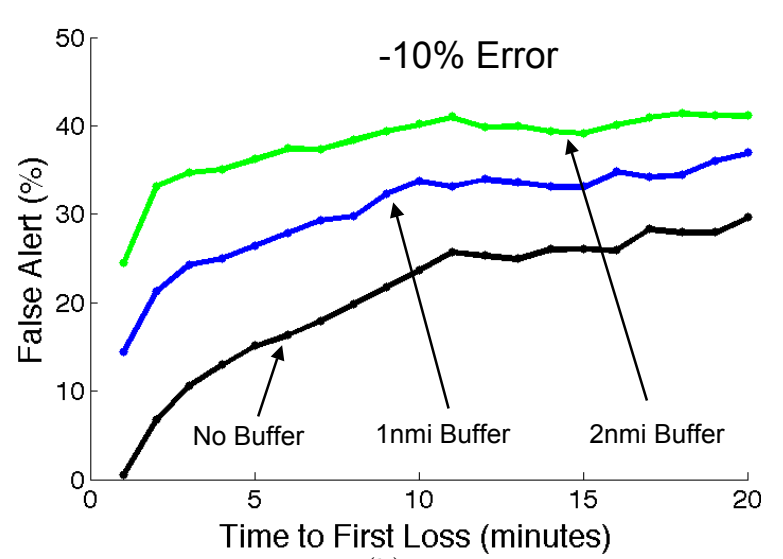

(b)

Figure 7. The percentage of incorrect conflict alerts for different buffer sizes as functions of the time to first loss for (a) $-5 \%$ speed error and (b) $-10 \%$ speed error.

and the speed error. The data are symmetric between positive and negative speed perturbations. Figure $8(\mathrm{~b})$ indicates that the total percentage of false alerts is at a relatively high value of approximately $30 \%$, but that this is not a strong function of the time to first loss or the speed error.

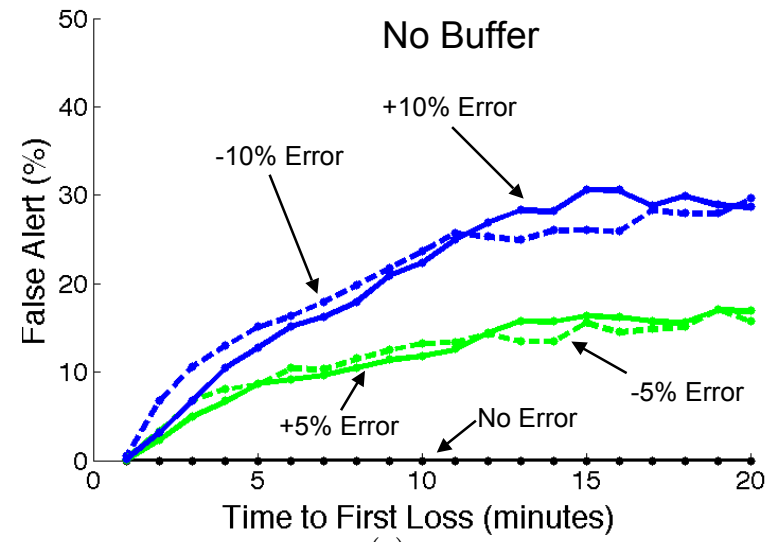

(a)

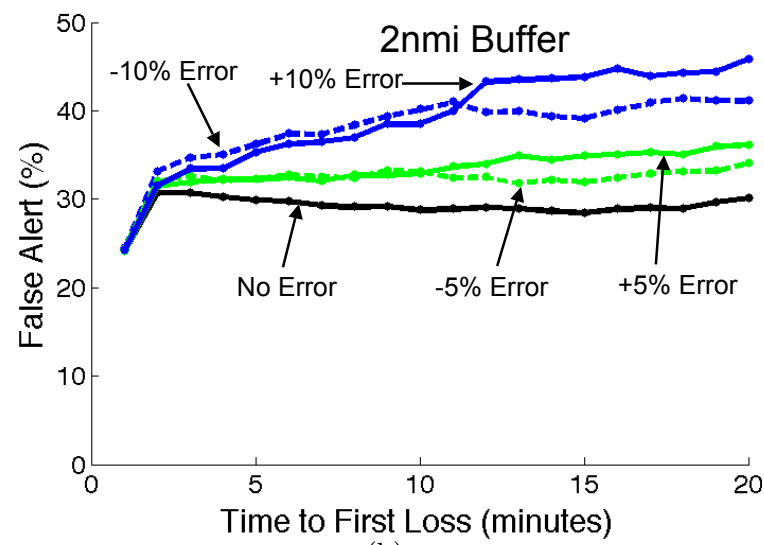

(b)

Figure 8. The percentage of incorrect conflict predictions for different speed errors as functions of the time to first loss for (a) no conflict detection buffer and (b) a 2 nmi detection buffer.

As shown by the previous results, increasing the detection buffer results in fewer missed alerts, but there are also many more false alerts for larger detection buffers. To better explain these trade-offs, the missed and false alert percentages are shown on the same plot in Figure 9 as functions of the conflict detection buffer. For two to four minutes before loss (Figure $9(\mathrm{a})$ the false alerts are a strong function of the detection buffer size, but the missed alerts are not. Both the false and missed alerts are largely insensitive to the speed error. For eight to ten minutes before loss of separation (Figure 9(b) though, the missed and false alerts are both strong functions of both the speed error and the detection buffer size. For a buffer size of 2 nmi there is approximately the same percentage of false alerts as for two to four minutes before loss, but there are many more missed alerts.

\section{B. Conflict Resolution}

For the conflict resolution study the conflict detection buffer was held constant at 1 nmi. The only data provided to the Autoresolver were the perturbed trajectory predictions. The simulated aircraft would fly the maneuvers provided by the Autoresolver based on the unperturbed data. The main independent variable in this study was the conflict resolution buffer size. 


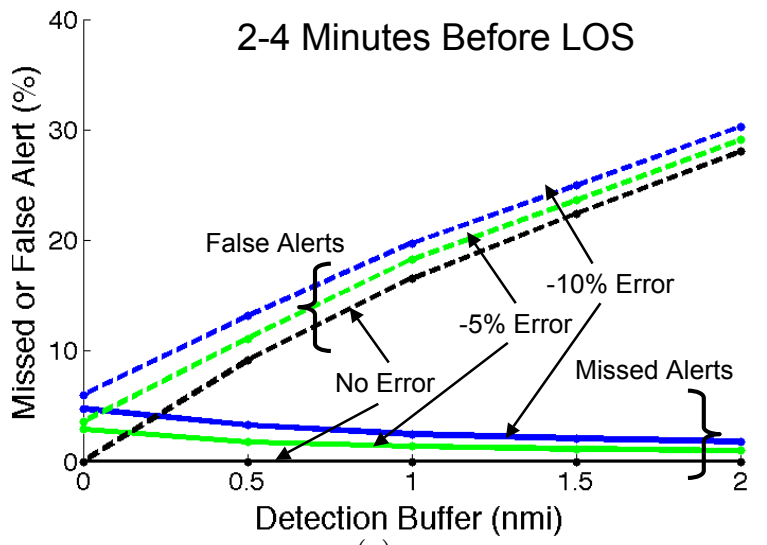

(a)

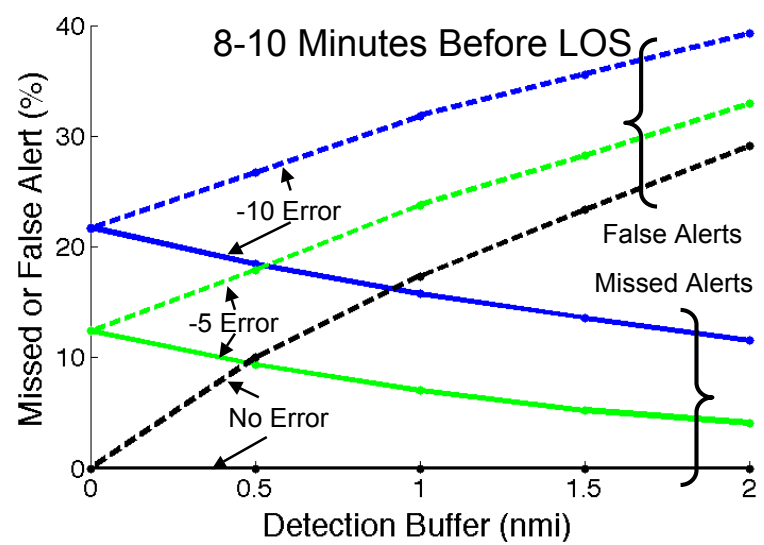

(b)

Figure 9. The missed and false alert percentages for different speed errors as functions of the conflict detection buffer size for all conflicts (a) two to four minutes from loss of separation and (b) eight to ten minutes before loss of separation.

Figure 10(a) shows the total number of resolutions performed by the Autoresolver for different speed errors as functions of the resolution buffer size. The total number of resolutions increases significantly with the trajectory prediction error and is symmetric between positive and negative errors. For all error levels, the total number of resolved conflicts decreases as a function of the resolution buffer size indicating that the resolution buffer performed its intended function of reducing the number of conflicts which need to be resolved twice.

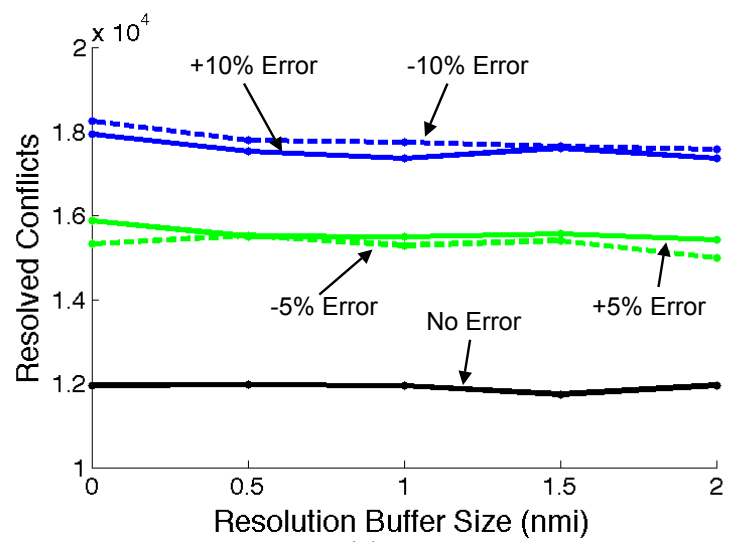

(a)

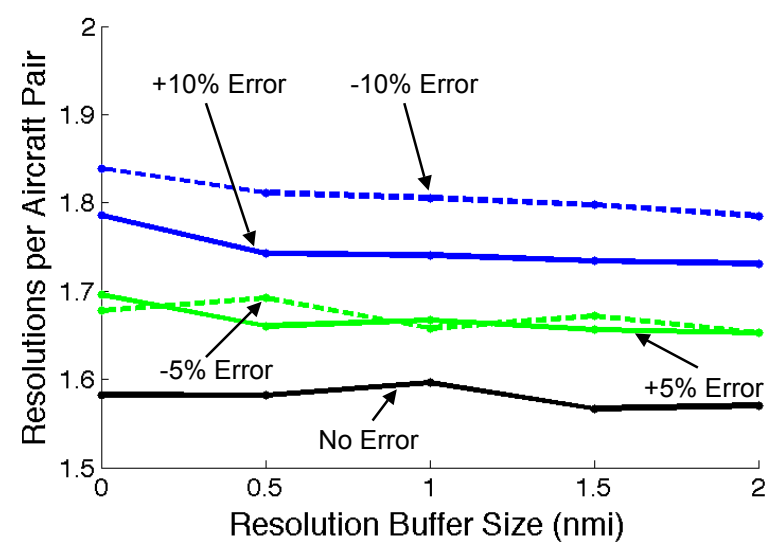

(b)

Figure 10. (a) The total number of resolutions and (b) the average number of resolutions per aircraft pair for different speed errors as functions of the conflict resolution buffer size.

The average number of resolutions for and given pair of aircraft experiencing a conflict is shown in Figure 10(b) This average number of resolutions is a fairly strong function of the resolution buffer size further indicating that the resolution buffer helped to prevent aircraft in conflict from becoming in conflict again due to trajectory speed errors.

The average delay per resolution and the total delay are shown in Figures 11(a) and 11(b) respectively. The average delay is a fairly strong function of the resolution buffer size because larger buffers require more delays. The total delay is not as dependent on the resolution buffer size because a larger resolution buffer offsets the increase in average delay by reducing the likelihood of having to recompute the resolution. Interestingly the delay was not symmetric between positive and negative speed errors. Positive speed errors tended to result in much higher average and total delay.

Further study of the geometric properties of these conflicts revealed that this was due to the angle of incidence of the conflict, where the angle of incidence of a head-on collision is $180^{\circ}$ and the angle of incidence 


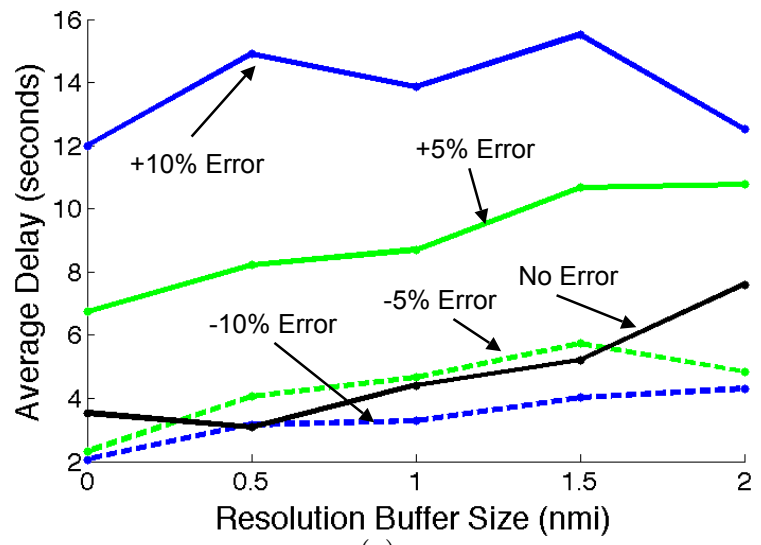

(a)

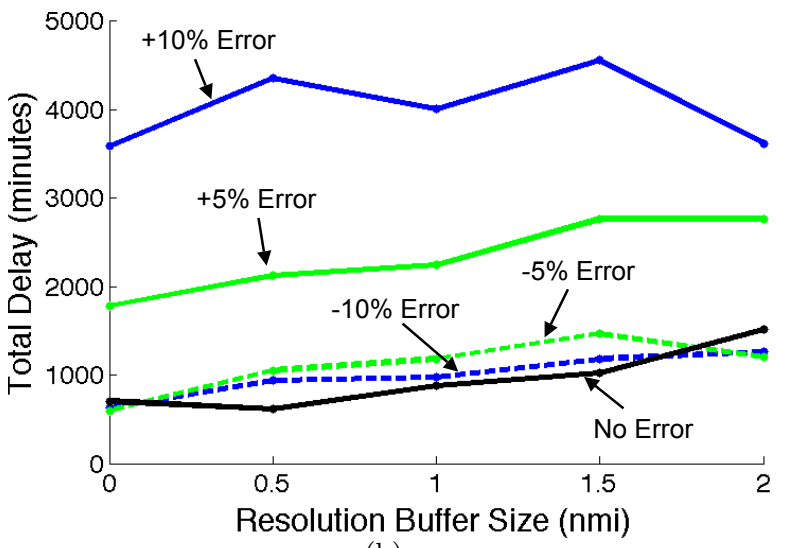

(b)

Figure 11. (a) The average delay and (b) the total delay for different speed errors as functions of the conflict resolution buffer size.

of an in-trail conflict is $0^{\circ}$. Figure $12(\mathrm{a})$ shows that for conflicts with an angle less than $90^{\circ}$ there are large differences between the average delays with positive errors and the average delays with negative errors. Figure 12(b) shows these differences are not present for conflicts with an angle of incidence greater than $90^{\circ}$.

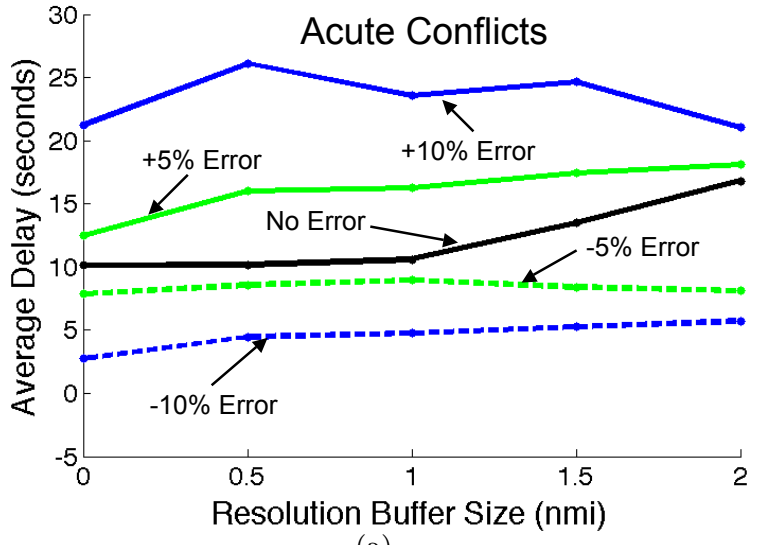

(a)

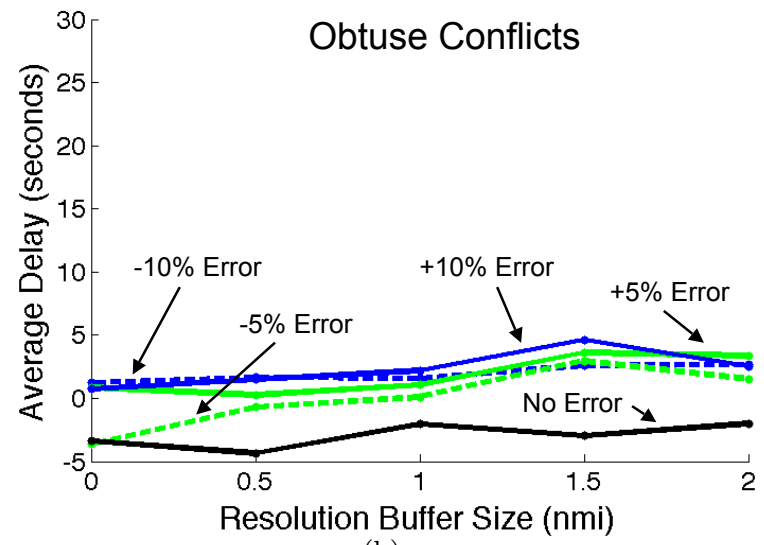

(b)

Figure 12. The total delay for all conflicts with an angle of incidence (a) less than or equal to $90^{\circ}$ and $(\mathrm{b})$ greater than $90^{\circ}$ for different speed errors.

Finally, the actual flown tracks were analyzed for losses of separation. Figure 13 shows the total number of losses of separation for the different speed errors. As was expected, this number was independent of the conflict resolution buffer. The no error case had losses of separation because of aircraft entering the simulation in conflict at either an international boundary or through an airport boundary. Figure 13 illustrates that the number of losses of separation increased with the magnitude of the speed error. This may be caused by the uncertainty in the time of top of descent caused by the speed errors. The relatively larger number of losses of separation in the $+10 \%$ case are due to the number of out-of-envelope trajectory-generation errors. These results indicate that either a larger conflict detection buffer or another method of mitigating the trajectory prediction errors, possibly including vertical buffers for climbing and descending aircraft, may be required.

\section{Conclusions}

This paper presented the results of a parametric study designed to determine the effects of aircraft speed errors on a separation assurance algorithm. The study was performed using a non-real time simulation environment which allowed for accurate knowledge of how the trajectories would be flown without any speed 


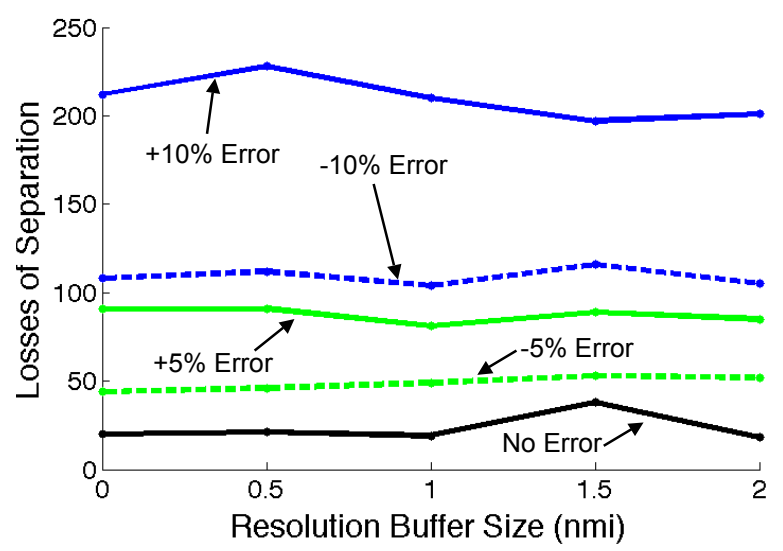

Figure 13. The total number of losses of separation for different speed errors.

errors. This accurate knowledge of the unperturbed trajectory allowed for a clear definition of both missed and false alerts.

The effects of speed errors on conflict detection accuracy were shown in relation to the additional buffer used for conflict detection above the required horizontal separation. For a speed error of $-10 \%$ of the actual cruise speed, the percentage of missed alerts at ten minutes before loss of separation was reduced from about $25 \%$ to about $12 \%$ by adding a $2 \mathrm{nmi}$ detection buffer. However, the same buffer increased the false alerts from about $20 \%$ to about $40 \%$, illustrating the expected trade-offs between false and missed alerts with respect to the conflict detection buffer.

Greater speed errors tended to increase the number of resolutions required to maintain separation of aircraft, and they increased the system-wide delay. Interestingly, positive speed errors increased the system delay significantly more than negative speed errors even though the magnitude of the error was the same. This seemed to be caused by an increase in the resolution delay required for in-trail type conflicts at higher cruising speeds. Increasing the resolution buffer size decreased the total number of resolutions, but it also increased the average delay per resolution.

Future work will expand this parametric study of trajectory prediction uncertainty from only focusing on speed errors to incorporating other types of error such as aircraft weight errors, top-of-descent point errors, descent profile errors, and maneuver implementation delays. Also, the efficacy of additional trajectory uncertainty mitigation steps will be studied.

\section{Acknowledgments}

The author would like to thank Tony Wang of the University-Affiliated Research Center for providing invaluable software development and support for this experiment. He would also like to thank the NASA reviewers of this paper that provided many helpful suggestions.

\section{References}

\footnotetext{
${ }^{1}$ Cone, A. and Chin, D., "Sensitivity of an Automated Separation Assurance Tool to Trajectory Uncertainty," AIAA Aviation Technology, Integration, and Operations Conference, No. AIAA 2009-7014, Hilton Head, South Carolina, 2009.

${ }^{2}$ Green, S. M. and Vivona, R., "Field Evaluation of Descent Advisor Trajectory Prediction Accuracy," AIAA Guidance, Navigation and Control Conference, No. AIAA-96-3764, 1996.

${ }^{3}$ Stell, L. L., "Predictability of Top of Descent Location for Operational Idle-Thrust Descent," AIAA Aviation Technology, Integration, and Operations Conference, Fort Worth, Texas, 2010.

${ }^{4}$ Green, S. M., "Descent Advisor Preliminary Field Test," AIAA Guidance, Navigation and Control Conference, Baltimore, Maryland, 1995.

${ }^{5}$ Bilimoria, K. D., "Methodology for the Performance Evaluation of a Conflict Probe," Journal of Guidance, Control, and Dynamics, Vol. 24, 2001, pp. 444-451.

${ }^{6}$ Erzberger, H., Paielli, R. A., Isaacson, D. R., and Eshow, M. M., "Conflict Detection and Resolution in the Presence of Prediction Error," 1st USA/Europe Air Traffic Management RED Seminar, Saclay, France, 1997.

${ }^{7}$ Meyn, L., Windhorst, R., Roth, K., Drei, D. V., Kubat, G., Manikonda, V., Roney, S., Hunter, G., and Couluris, G.,
} 
"Build 4 of the Airspace Concepts Evaluation System," AIAA Modeling and Simulation Technologies Conference and Exhibit, No. AIAA 2006-6110, 2006.

${ }^{8}$ Erzberger, H., "Transforming the NAS: The Next Generation Air Traffic Control System," NASA/TP-2004-212828, 2006.

${ }^{9}$ Erzberger, H., Lauderdale, T. A., and Cheng, Y., "Automated Conflict Resolution, Arrival Management and Weather Avoidance for ATM," 27th Iternational Congress of the Aeronautical Sciences, Nice, France, 2010.

${ }^{10}$ Erzberger, H., "Automated Conflict Resolution for Air Traffic Control," 25th Iternational Congress of the Aeronautical Sciences, 2006.

${ }^{11}$ Farley, T. C. and Erzberger, H., "Fast-Time Simulation Evaluation of a Conflict Resolution Algorithm Under High Air Traffic Demand," 7th USA/Europe ATM 2007 REDD Seminar, 2007.

${ }^{12}$ McNally, D. and Thipphavong, D., "Automated Separation Assurance in the Presence of Uncertainty," 26th International Congress of the Aeronautical Sciences, 2008.

${ }^{13}$ Thipphavong, D., "Analysis of Climb Trajectory Modeling for Separation Assurance Automation," AIAA Guidance, Navigation, and Control Conference, No. AIAA 2008-7407, 2008.

${ }^{14}$ Prevot, T., Homola, J., Mercer, J., Mainini, M., and Cabrall, C., "Initial Evaluation of Air/Ground Operations with Ground-Based Automated Separation Assurance," 8th USA/Europe Air Traffic Management RESD Seminar, Napa, California, 2009.

${ }^{15}$ Thipphavong, D., "Analysis of a Multi-Trajectory Conflict Detection Algorithm for Climbing Flights," AIAA Aviation Technology, Integration, and Operations Conference, No. AIAA 2009-7021, 2009.

${ }^{16}$ Schwartz, B. E., Benjamin, S. G., Green, S. M., and Jardin, M. R., "Accuracy of RUC-1 and RUC-2 Wind and Aircraft Trajectory Forecasts by Comparison with ACARS Observations," Weather and Forecasting, Vol. 15, No. 3, 2000 , pp. 313-326. 